\title{
Could the neuroprotective drug NA-1 limit ischaemic brain damage after stroke?
}

Despite considerable success in preclinical studies, pharmacological neuroprotective strategies to minimize ischaemic brain damage after stroke have largely failed to translate to the clinic. The results of a new phase II trial published in The Lancet Neurology, however, indicate potential benefits of the neuroprotective drug NA-1 when administered in the immediate aftermath of a stroke.

NA-1 is an inhibitor of the postsynaptic scaffolding protein PSD-95, which is involved in NMDA receptor-mediated excitotoxicity. The drug has previously been shown to have neuroprotective effects in both rodent models and oldworld primates.

"Stroke is a death knell for any drug company at the moment-too many trial failures," explains Michael Hill from the University of Calgary, AB, Canada, who led the new study. "We used a novel paradigm for testing a neuroprotective drug: iatrogenic strokes occurring during aneurysm repair and assessed using a surrogate outcome measure on MRI."

\section{Stroke is a death knell} for any drug company at the moment... 77

The ENACT (Evaluating Neuroprotection in Aneurysm Coiling Therapy) trial recruited 185 individuals who were about to undergo endovascular treatment to repair a ruptured or unruptured intracranial aneurysm - a procedure that carries a high risk of small ischaemic strokes. The participants were randomly assigned to receive an intravenous infusion of NA-1 $(n=92)$ or saline $(n=93)$ at the end of the endovascular procedure. Brain MRI was used to assess the number and volume of new ischaemic strokes sustained during the procedure.

Around two-thirds of the total study population experienced small ischaemic strokes during endovascular treatment. No differences in infarct volumes were observed between the saline and NA-1 groups; however, the patients treated with NA-1 showed significantly fewer new brain lesions, on average, than those who received saline.

According to Hill, the results provide "clear proof that neuroprotection in humans is possible." The researchers now plan to examine whether administration of NA-1 in the ambulance environment can improve outcomes in patients with community-onset stroke.

Heather Wood

Original article Hill, M. D. et al. Safety and efficacy of NA-1 in patients with iatrogenic stroke after endovascular aneurysm repair (ENACT): a phase 2, randomised, double-blind, placebo-controlled trial. Lancet Neurol. 11, 942-950 (2012) Further reading Cook, D. J. et al. Treatment of stroke with a PSD-95 inhibitor in the gyrencephalic primate brain. Nature 483, 213-217 (2012) 